\title{
Estudio de la internacionalización de la Universidad Nacional de Mar del Plata desde la perspectiva de la producción científica Análisis de la colaboración y liderazgo
}

\section{Gustavo Liberatore}

Universidad Nacional de Mar del Plata. Facultad de Humanidades. Depto. Ciencia de la Información I gliberat@mdp.edu.ar / https://orcid.org/oooo-0002-5841-8739

\section{Silvia Sleimen}

Universidad Nacional de Mar del Plata. Facultad de Humanidades. Depto. Ciencia de la Información | sislei@mdp.edu.ar / https://orcid.org/oooo-0001-6378-7304

\begin{abstract}
Andrés Vuotto
Universidad Nacional de Mar del Plata. Facultad de Humanidades. Depto. Ciencia de la Información | avuotto@gmail.com / https://orcid.org/oooo-0002-1897-9515

\section{Victoria Di Césare}

Universidad Nacional de Mar del Plata. Facultad de Humanidades. Depto. Ciencia de la Información I vdicesare@mdp.edu.ar / https://orcid.org/oooo-ooo1-7098-1914
\end{abstract}

\section{Natalia Pallotta \\ Universidad Nacional de Mar del Plata. Facultad de Humanidades. Depto. Ciencia de la Información| npallotta@mdp.edu.ar / https://orcid.org/oooo-0002-0137-2530}

\section{Resumen}

En este trabajo se exponen los resultados obtenidos del análisis y caracterización de la internacionalización de la actividad científica de la Universidad Nacional de Mar del Plata (UNMDP) a partir del estudio de la producción publicada desde su creación. La investigación abarca el período 1978-2019 y está centrada en los artículos publicados durante dicho lapso. Las fuentes secundarias utilizadas fueron Web of Science Core Collections (Clarivate) y Scopus (Elsevier) de las que se recuperaron los registros que conforman el objeto de estudio. Para el análisis de esta dimensión fueron aplicados los indicadores de colaboración autoral (instituciones y países), colaboración por grandes áreas temáticas, liderazgo científico e idioma de publicación. Además de los resultados y las respectivas representaciones de los indicadores propuestos se desarrollan algunas reflexiones sobre la medición de la internacionalización en instituciones de educación superior y los desafíos que presenta para una institución universitaria.

\section{Palabras clave}

Internacionalización Producción científica Universidad Nacional de Mar del Plata

Análisis bibliométrico 
Keywords

Internationalization Scientific production Universidad Nacional de Mar del Plata Bibliometric analysis

\title{
Study of the internationalization of the Universidad Nacional de Mar del Plata from the scientific production perspective: collaboration and scientific leadership analysis
}

\begin{abstract}
This work presents the results obtained from the internationalization analysis and characterization of Universidad Nacional de Mar del Plata (UNMDP) scientific activity, from the study of the scientific production published since its creation. The research covers the period 1978-2019 and is centered on the papers published during that time. The secondary sources used were Web of Science Core Collections (Clarivate) and Scopus (Elsevier), from which the records that form the subject of study were recovered. For the analysis of this dimension, indicators such as author collaboration (institutions and countries), collaboration by thematic areas, scientific leadership and publication language were applied. In addition to the results and representations of the proposed indicators, some considerations about the internationalization measurement within upper education institutions are made.
\end{abstract}

Artículo recibido: 18-09-2020. Aceptado: 9-04-2021

\section{Introducción ${ }^{1}$}

1. Los resultados de esta investigación fueron publicados en formato preprint en el repositorio ESOCITE. Disponible en https:// repositorio.esocite.la/503/1/Internacionalizacion_preprint.pdf
Las universidades nacionales públicas argentinas constituyen un eslabón muy importante en el sistema científico y tecnológico nacional aportando el porcentaje más alto de la producción científica registrada, muy por encima de otros organismos nacionales de ciencia y tecnología (CyT) y de instituciones privadas del sector. Históricamente, ha sido dificultoso acceder a estadísticas oficiales sobre indicadores fiables y actualizados del desempeño de las actividades científicas y tecnológicas (ACyT) en su conjunto $\mathrm{y}$, en particular, de las producciones que esta genera. En la actualidad, los únicos dispositivos de memoria a nivel nacional consagrados al registro individual de las ACyT como el Sistema Integral de Gestión y Evaluación (SIGEVA) o el Registro Unificado y Normalizado de Datos Curriculares del personal científico y tecnológico (CVar) (este último en un estado de virtual parálisis) son de difícil acceso debido a medidas restrictivas de las instituciones que los gestionan, sumado a un diseño de la matriz de datos poco funcional y clara. Frente a este escenario, la capacidad de acceso de las instituciones de educación superior a los datos de sus propios registros sobre las diferentes ACyT $\mathrm{y}$, en particular, de las producciones intelectuales derivadas de ellas es limitada.

En este contexto se hace visible la necesidad de desarrollar instrumentos y metodologías que permitan afrontar, por un lado, la carencia de fuentes secundarias confiables y actualizadas que recojan las producciones intelectuales de las instituciones científicas y académicas nacionales y, por el otro, abordar investigaciones empíricas basadas en datos confiables y representativos. Como respuesta a esta problemática, en la Universidad Nacional de Mar del Plata (UNMDP) se encuentra en desarrollo el "Observatorio de la producción científica de la UNMDP" creado con el objetivo de constituir un dispositivo que se oriente a la provisión de insumos (datos/indicadores) para la gestión, evaluación y difusión de las actividades científicas y académicas de la institución. Esta investigación constituye el primer producto de este observatorio, obtenido a través de los datos disponibles en bases de datos de corriente principal e indaga sobre las características del proceso de internacionalización de la UNMDP a partir del análisis de su producción intelectual. En particular, este trabajo intenta reflejar de manera estructural cuáles son las áreas de conocimiento involucradas en este fenómeno y en qué medida el componente "internacionalización" ha ido 
evolucionando a lo largo de la historia institucional. De forma específica, se observa cuáles son los principales países con los que se han desarrollado vínculos de colaboración y las instituciones involucradas. Por último, se analiza el nivel de liderazgo que la UNMDP desempeña en los procesos de intercambio dentro del plano internacional.

\section{Acerca de los procesos de internacionalización en la educación superior}

El término internacionalización constituye un genérico que se aplica a diversos ámbitos y su uso ha ganado fuerza, desde los años 90 , no solo como consecuencia del desarrollo de la denominada Sociedad de la información, en un sentido de época, sino también por razones tecnológicas, políticas y por ambas, combinadas en modo sinérgico.

La internacionalización en los espacios académicos ha recorrido y aún recorre diferentes terrenos, todos ellos interconectados, en los que la formación de posgrado, la movilidad de docentes e investigadores y la producción científica podrían señalarse como los principales.

Desde un punto de vista estructural y desde una perspectiva política, frente a la disponibilidad de recursos provenientes de las tecnologías de la información y la comunicación (TICs), de políticas científicas sumamente ambiciosas y de presupuestos generalmente estables o con mínimos altibajos, los grandes espacios académicos y de investigación de los países centrales fueron acentuado su centralidad y fortaleciendo sus posiciones dominantes en las tres actividades mencionadas. En tal sentido, las preguntas que América Latina se formuló desde fines de la década del '50, con la creación de la Comisión Económica para el Desarrollo de América Latina y el Caribe (CEPAL) y el pensamiento latinoamericano en Ciencia, Tecnología y Sociedad (CTS), giraron en torno a los nuevos desafíos que planteaba este escenario.

En una observación sencilla se advierte que los países latinoamericanos continúan operando de acuerdo con condiciones desventajosas de producción de carácter histórico y que llevan a la necesidad de pensar en conjunto si es posible deshacerse de dichas condiciones, generar parámetros comportamentales nuevos y alianzas regionales sostenidas, que redunden en beneficios al conjunto, a sus instituciones y a las comunidades científicas involucradas. Esta independencia incluye, por supuesto, las agendas de investigación.

En esa dirección las conferencias de Educación Superior en América Latina y el Caribe celebradas los últimos años se conformaron en un espacio de pensamiento regional cuyas declaraciones han ido guiando el camino. Es así que la "Declaración de Cartagena" de 2008 (Conferencia Regional de Educación Superior [CRES], 2008) renovó el desafío de considerar a la educación superior como un bien social en sintonía con las enormes desigualdades existentes en el subcontinente. Plasmó además, consignas tales como la necesidad de constitución y fortalecimiento de redes regionales, formulación de políticas que eviten la emigración de jóvenes calificados hacia los países centrales y el requerimiento de constituir un "Espacio de Encuentro Latinoamericano y Caribeño de Educación Superior (ENLACES)" que va a ir tomando forma en los años siguientes. En 2014, ENLACES constituyó grupos de trabajo en busca de la redacción de agendas propias capaces de elaborar "orientaciones políticas en cuanto a cooperación, integración, e internacionalización”" .

2. http://espacioenlaces. org/antecedentes/

La tarea prosiguió en la Conferencia Regional de Educación Superior (CRES) de 2018, celebrada en Córdoba en el centenario de la Reforma Universitaria de 1918 en la que la internacionalización e integración de América Latina y el Caribe, constituyó uno 
de sus siete ejes de trabajo (Diker, 2018). Desde la base de los sistemas, en tanto, los investigadores latinoamericanos han buscado en todo momento en forma individual y/o grupal su crecimiento académico y el aprovechamiento de becas y otros estímulos que posibilitaran su formación cuaternaria, su participación en grupos de investigación con proyección internacional y el financiamiento de proyectos de investigación por parte de instituciones y organismos multilaterales o internacionales. La inestabilidad política, las deficiencias presupuestarias y la carencia o debilidad de políticas de Estado que estimulen la promoción científica, han constituido y constituyen en numerosos casos el contexto en el que desarrollan sus trabajos, con lo que la búsqueda de fuentes / recursos / oportunidades en los países centrales impide el desarrollo de agendas propias, independientes y con fuerte arraigo en las necesidades, problemas y oportunidades de este subcontinente.

Aparecen así una serie de elementos contextuales, de naturaleza política, científica, económica, institucional, que deben aplicarse en la búsqueda de respuestas a preguntas tales como qué se investiga, cómo se produce, en qué formatos, quién evalúa, qué méritos se atribuyen a la producción desde las instancias de evaluación científica, entre otras importantes cuestiones inherentes a la internacionalización, considerada en este ámbito.

\section{Análisis de los antecedentes desde las métricas de la internacionalización}

Para analizar los procesos de internacionalización de las instituciones científicas y académicas es preciso observar datos empíricos de la realidad que reflejen la dinámica de las distintas variables que componen este fenómeno. Frente a la necesidad de contar con instrumentos fiables para medir, evaluar y comparar las diferentes trayectorias institucionales y/o nacionales se han ido desarrollando sistemas de indicadores que contribuyeron a la consolidación de diferentes métricas de la internacionalización (Sebastián, 2011). De los instrumentos utilizados internacionalmente se destaca en primer lugar el Manual de Frascati en su versión de 2015, diseñado para países miembros de la Organización para la Cooperación y el Desarrollo Económicos (OCDE), que desarrolla un capítulo orientado a la medición de la "Globalización de la I+D" (OCDE, 2015: 297) con un marcado sesgo sobre la dimensión económica de las actividades científicas y tecnológicas (Michelini, 2018). Desde una perspectiva regional y enfocada no solo en los sistemas nacionales de ciencia y tecnología sino también en las instituciones involucradas en las actividades de I+D, el Manual de Santiago (Red Iberoamericana de Indicadores de Ciencia y Tecnología [RICYT], 2007) proporciona una serie de indicadores destinados a representar la heterogeneidad de países con sistemas periféricos o de mayor debilidad en términos de desarrollo científico (Sebastián, 2010). Estos estándares, no obstante, proveen una herramienta de medición sujeta a continuos debates ya que la aplicación práctica de los indicadores está condicionada a los diferentes contextos científicos y tecnológicos (institucionales, nacionales, regionales) desde donde son elaborados los informes. Se añade, además, que en regiones como Latinoamérica los datos necesarios para el cálculo de los indicadores no siempre resultan accesibles de manera completa o con la calidad requerida debido a deficiencias propias de los sistemas de información en CyT o a barreras de acceso impuestas por servicios externos (suscripción a bases de datos, por ejemplo). Finalmente, existen algunas diferencias relacionadas con el abordaje del fenómeno de la internacionalización y su forma de evaluación. Como expone Michelini (2018), los procesos de internacionalización de la ciencia y de internacionalización de la educación superior constituyen fenómenos diferentes, pero relacionados en función de sus actores, contextos y resultados. 
Desde el enfoque bibliométrico existen una batería de indicadores que son utilizados para medir la internacionalidad o grado de apertura de diferentes ámbitos e instrumentos de comunicación y producción científicos a partir de los datos obtenidos de las publicaciones. Al igual que los sesgos atribuidos a los instrumentos mencionados en el párrafo anterior, el grupo de indicadores bibliométricos utilizados en la medición de la internacionalización o globalización de la ciencia no están exentos de diferencias de interpretación dependiendo del contexto político desde donde se los aplique como dispositivo de evaluación de estos fenómenos (Moed, 2017). Como bien apuntan Robinson-García y Ràfols (2020) existe una tendencia a una "interpretación universal" de los resultados obtenidos con los indicadores bibliométricos cuando en realidad los datos aportados por estas metodologías de análisis dependen muy a menudo de los contextos en donde son aplicados. Así, las culturas evaluativas instaladas en los sistemas científicos de países centrales basadas esencialmente en el recuento de citas y la valoración de las fuentes de publicación (revistas) en función de una medida de impacto suelen no ser los parámetros más adecuados de evaluación en sistemas científicos menos desarrollados o con metas de circulación del conocimiento diferentes (Beigel, 2013).

Los estudios bibliométricos orientados a la dimensión de la internacionalización suelen pendular entre aquellos meramente descriptivos y las investigaciones más analíticas destinadas a poner de relieve aspectos más complejos. Por lo general, los ámbitos cubiertos se ubican en tres posibles escenarios: las culturas de producción y sus fuentes de publicación, las instituciones y los sistemas nacionales de I+D, las dinámicas propias de los campos disciplinares y los patrones de citación. Dentro de los múltiples abordajes de estas facetas existen algunos que aportan nuevas perspectivas para el estudio de este fenómeno, como es el caso de Schubert y Schubert (2020) que proponen dos nuevas dimensiones para el análisis como son la "profundidad" medida en número de publicaciones y citas internacionales y la "amplitud" en la relación con la cantidad de países colaborantes.

El tema de la colaboración como característica distintiva de las actividades científicas y sus modos de producción ha generado estudios ya clásicos (Beaver y Rosen, 1979; Katz y Martin, 1997; Sonnenwald, 2007) dentro de las referencias utilizadas en los estudios métricos de la información. En tanto, son múltiples los trabajos publicados sobre los patrones de publicación científica, el análisis de la coautoría y las características de la colaboración de los cuales se citan aquí algunos ejemplos (Escribà-Sales y Cortiñas, 2013; Jung y Ruiz-León, 2018; Leydesdorff et al., 2013; Mindeli y Markusova, 2015).

Otro tanto ocurre con los estudios de la internacionalización de las revistas como principal vehículo de la difusión de la producción científica (López-Robles et al., 2019; Navas-Fernández, Abadal y Rodrigues, 2018; Pandiella, García-Zorita y SanzCasado, 2019). En estos casos, las variables de análisis se centran en los factores que, se presume, inciden en el nivel de participación extranjera en las publicaciones. Aunque no existen evidencias concluyentes, el idioma, el nivel de visibilidad de la fuente, la conformación de los comités editoriales y el área disciplinar de pertenencia se encuentran entre los principales aspectos que impactan en esta dimensión.

Los procesos de internacionalización de las instituciones de educación superior también han sido objeto de estudio desde abordajes métricos (Oliveira et al., 2020; De Filippo, Marugán y Sanz-Casado, 2014; Sleimen, 2015; Souza, De Filippo y SanzCasado, 2019). En general, y particularmente en los antecedentes aquí citados, la medida de internacionalización se funda en el grado de apertura de la producción generada por instituciones científicas y universitarias como reflejo de sus políticas y programas de inserción en publicaciones de corriente principal. En cierto sentido, la observación de este fenómeno desde sistemas institucionales de educación superior 
3. Código disponible en: https://github.com/ VictoriaDiCesare/R-Code/blob/ master/clean-char-strings.R pertenecientes a países por fuera de la élite de naciones desarrolladas parece ser un objetivo "necesario" para medir su grado de contacto y colaboración en ámbitos de la denominada "buena ciencia". Esto explica, en parte, por qué la mayoría de estas investigaciones se basan en datos provenientes de las dos bases de datos internacionalmente representativas del "mainstream" científico como son Web of Science y Scopus. Desde otra perspectiva, es también cierto que estas son las fuentes secundarias que casi exclusivamente aportan los datos necesarios para medir con mucha precisión las distintas variables que, en términos bibliométricos, caracterizan los procesos de internacionalización.

Las culturas de producción asociadas a las características intrínsecas de algunas áreas de conocimiento son también abordadas desde la perspectiva de la internacionalización. En particular, las ciencias sociales y humanidades han sido objeto de estudio en términos de los flujos de colaboración y los patrones de citación observados de cara a procesos de globalización crecientes. La observación de este fenómeno se encuentra en constante debate (Laclette et al, 2011; Mosbah-Natanson y Gingras, 2014), tanto desde aquellos enfoques basados en el modelo centro/periferia cuanto desde los que analizan la internacionalización de la producción en estos campos bajo los límites de una centralidad relativa a la investigación situada y de características moldeadas por el contexto.

\section{Material y métodos}

El universo de análisis de esta investigación está comprendido por los artículos publicados por investigadores de la UNMDP en revistas de corriente principal indizadas en las bases de datos Web of Science Core Collection (Science Citation Index, Social Science Citation Index, Arts \& Humanities Citation Index y Emerging Sources Citation Index) y Scopus. El período de estudio está comprendido desde el año en que aparece indizada la primera publicación con autores de esta institución hasta la actualidad, conformando un lapso comprendido entre los años 1978-2019. Es importante destacar que la ventana de tiempo resultante es casi equivalente a la antigüedad de esta institución ya que su constitución como universidad nacional data de 1975 .

Las búsquedas se realizaron entre los meses de febrero y marzo de 2020 utilizando las formas de descripción institucional normalizada que poseen ambas bases de datos, siendo para WoS el campo OG= Organización-Consolidada y para Scopus el identificador de afiliación AF-ID(60002864). Para aquellas instituciones distintas de la UNMDP derivadas de las filiaciones de los autores se aplicó un proceso de normalización manual.

El número total de ítems recuperados correspondientes al tipo documental "artículo" fue de 10847 registros, observándose un solapamiento entre ambas bases de datos del $76 \%$. El proceso para determinar este porcentaje de superposición de contenidos fue llevado a cabo en $R$. El procedimiento elegido consistió en reducir los títulos de las publicaciones a cadenas de caracteres en minúscula, sin espacios y sin signos de puntuación para posibilitar tanto su comparación, como la identificación y eliminación de los registros duplicados ${ }^{3}$.

Del total, fueron filtradas aquellas publicaciones en colaboración para analizar el componente internacionalización mediante la aplicación de un conjunto de indicadores. La caracterización de esta unidad de análisis comenzó con la delimitación de la filiación geográfica e institucional de los autores para determinar el grupo de publicaciones que fueron realizadas con colaboración extranjera. Para elaborar una mayor descripción, los registros se agruparon en cinco grandes áreas temáticas: Social 
Sciences (incluye las humanidades), Life Sciences, Physical Sciences, Health Sciences y Multidisciplinar. La decisión metodológica de utilizar grandes áreas se debió a que ambas bases de datos, WoS y Scopus, utilizan sistemas de clasificación difíciles de compatibilizar por lo que se optó por trabajar con grandes grupos temáticos que disminuyeran el sesgo implícito en cada una de las fuentes utilizadas. Dado que la unidad de clasificación es la revista, fueron respetados los solapamientos existentes entre las diferentes categorías temáticas de cada una de las bases de datos, ajustando finalmente el número de registros de cada área mediante una cuenta fraccionaria. Se calculó además el índice de coautoría (IC) discriminado por área temática para observar las diferencias entre los patrones de colaboración. El idioma de publicación fue otra variable de observación ya que constituye uno de los factores gravitantes en los procesos de internacionalización. Finalmente, se realizó un análisis de corte cualitativo aplicando el indicador de "liderazgo científico" propuesto por Moya-Anegón (MoyaAnegón, 2012; Jeremi et al., 2013) en el Scimago Institution Ranking (SIR) definido como el volumen de producción en el cual la institución es contribuyente, en este caso la UNMDP, determinada por la cantidad de artículos en el que el autor responsable pertenece a dicha entidad. Este dato se obtiene de la dirección de correspondencia fijada por el autor líder.

Para el almacenamiento y procesamiento de los datos se diseñó una base de datos ad hoc en el sistema MySQL. Para el cálculo de los indicadores y su representación se utilizaron las aplicaciones $R$ y Microsoft Excel. Las matrices de colaboración fueron representadas por grafos derivados de la aplicación del análisis de redes sociales (ARS). Para la valoración de los componentes de la redes se utilizaron medidas de centralidad (grado e intermediación) y de modularidad (análisis de comunidades o clusters). Para la visualización de datos, se ha utilizado el programa Gephi en su versión 0.9.2 (2017), seleccionando para la distribución el algoritmo ForceAtlas.

\section{Resultados y discusión}

La producción científica colaborada de la UNMDP ( $\mathrm{n}=8884$ ) registrada en la corriente principal representa el $86 \%$ de todas sus publicaciones $(\mathrm{N}=10847)$, mientras que de este conjunto el $38 \%$ ha sido producida con colaboración internacional ( $\mathrm{n}=3417)$ (Tabla 1). La distribución de este último porcentaje, cuando se lo calcula por las áreas temáticas seleccionadas, presenta importantes variaciones.

Tabla 1. Distribución de la producción científica colaborada

\begin{tabular}{|l|c|c|c|c|c|c|}
\hline Áreas temáticas & $\begin{array}{c}\text { Total } \\
\text { artículos }\end{array}$ & $\begin{array}{c}\text { Artículos en } \\
\text { colaboración }\end{array}$ & $\begin{array}{c}\% \\
\text { total de art. } \\
\text { en colab. }\end{array}$ & $\begin{array}{c}\% \\
\text { art. en colab. } \\
\text { por área }\end{array}$ & $\begin{array}{c}\text { Art. en } \\
\text { colab. int. }\end{array}$ & $\begin{array}{c}\% \\
\text { art. en } \\
\text { colab. Int. }\end{array}$ \\
\hline Physical Sciences & 4860 & 4357 & 49,04 & 89,65 & 1783 & 40,92 \\
\hline Life Sciences & 4179 & 3522 & 39,64 & 84,28 & 1225 & 34,78 \\
\hline Social Sciences & 952 & 530 & 5,97 & 55,67 & 141 & 26,60 \\
\hline Health Sciences & 779 & 425 & 4,78 & 54,56 & 268 & 63,06 \\
\hline Multidisciplinar & 77 & 50 & 0,56 & 64,94 & 0 & 0 \\
\hline TOTALES & $\mathbf{1 0 8 4 7}$ & $\mathbf{8 8 8 4}$ & $\mathbf{1 0 0}$ & & 3417 & \\
\hline
\end{tabular}




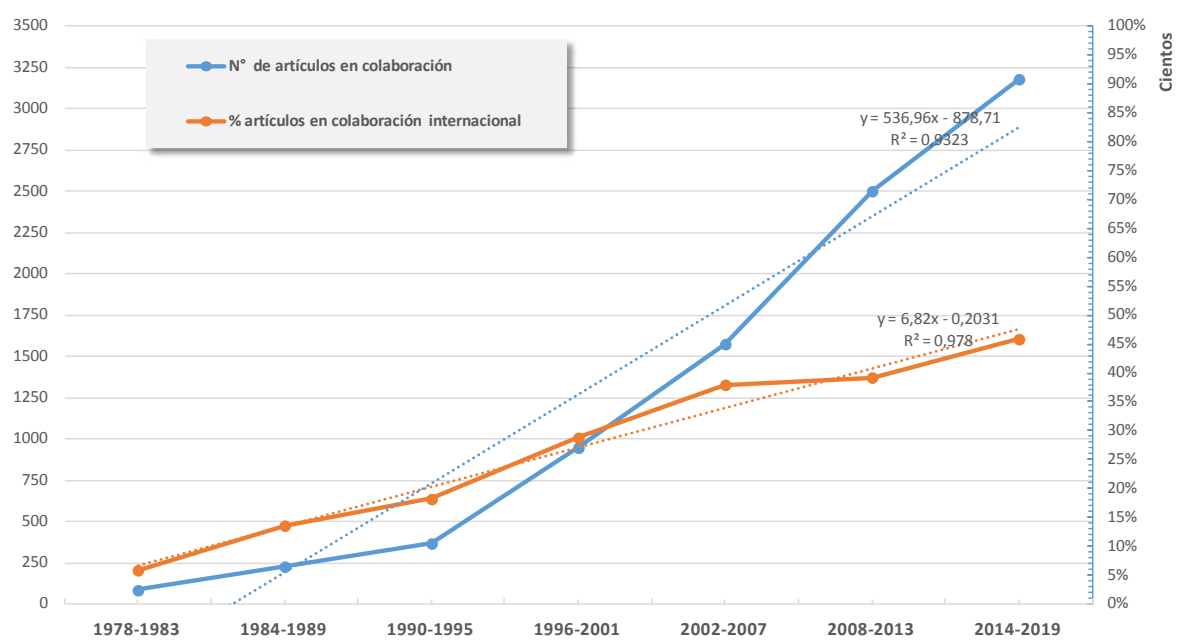

Figura 1. Evolución de la producción total colaborada y porcentaje de la participación extranjera

Los mayores porcentajes de colaboración corresponden a las áreas Physical Sciences y Life Sciences, sumando ambos casos casi el $90 \%$ de la producción total. También se desprende que casi la totalidad de las publicaciones de estos dos dominios $(89,65 \%$ y $84,28 \%$ respectivamente) generan una producción colaborada. Sin embargo, cuando se observa la participación internacional en esta variable por áreas temáticas se deduce que no existe una correspondencia directa entre los porcentajes generales de colaboración y los calculados para la colaboración internacional. Así, el área Health Sciences es la que presenta una mayor participación de autores extranjeros en las publicaciones (63,06\%), seguida por Physical Sciences (40,92\%), Life Sciences (34,78\%) y finalmente Social Sciences (26,6\%). Es importante advertir, no obstante, que el volumen de producción del área con mayor participación extranjera es mucho menor que el de las dos siguientes, por lo que no es posible ser concluyentes en términos de que estas proporciones constituyan un patrón. De igual manera debe valorarse, aunque en el sentido contrario, el área Social Sciences. La única área que no registra colaboración internacional es la Multidisciplinar por lo que, además de ser despreciable en el volumen de producción, no formó parte en el análisis de esta dimensión.

Cuando observamos la evolución de esta variable se aprecia que la participación extranjera ha estado en constante crecimiento, ubicándose en el último sexenio (Figura 1) en el orden del $46 \%$ del total de la producción colaborada.

Dentro de las características que adopta la autoría en los procesos de internacionalización puede apreciarse una constante en las cuatro áreas estudiadas respecto del indicador de coautoría. Los índices resultantes (IC) en los cálculos realizados demuestran en todos los casos que en las publicaciones con participación extranjera (ICI) el número de firmas es mayor que en los artículos en colaboración nacional (ICN) (Figura 2). Este aspecto reafirma la idea de la existencia de diferentes culturas de publicación cuando la producción deriva de un proceso de colaboración internacional, acentuándose la autoría múltiple producto de la integración de distintos equipos de investigación (Robinson-García y Amat, 2018). En particular, este rasgo se hace muy evidente en el área Social Sciences en donde la diferencia del IC entre ambas dimensiones, nacional y extranjera, es muy grande. 


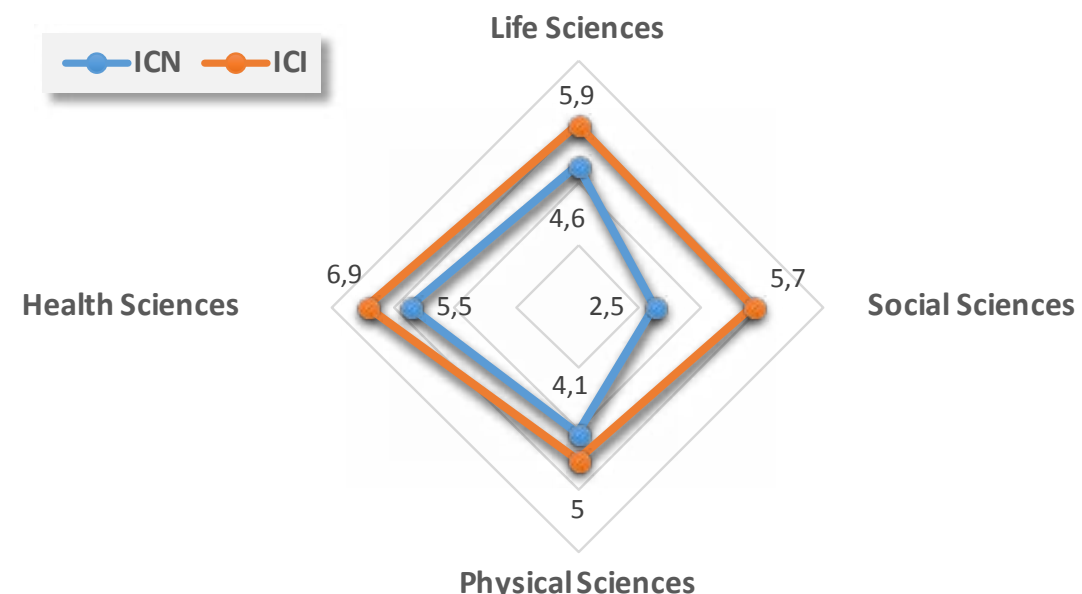

$\mathrm{ICN}=$ índice de colaboración nacional | ICl= índice de colaboración internacional.

Figura 2. Variaciones del índice de coautoría en relación con la producción colaborada por área temática

Desde una perspectiva global y en términos de las publicaciones científicas la UNMDP ha colaborado dentro del período estudiado, es decir, a lo largo de casi toda su trayectoria, con 85 países. El número resultante da a entender una gran apertura. Sin embargo, cuando se analiza dónde se concentran las mayores frecuencias de colaboración con autores de otras latitudes se comprueba que el 50\% de la producción colaborada internacionalmente se realiza con apenas 5 países, mientras que el $90 \%$ de las publicaciones se amplifica a un conjunto delimitado por solo 25 países (Tabla 2).

Al observar la lista puede advertirse la existencia de un núcleo principal de cinco países que configuran los rasgos que presenta la internacionalización de la investigación en la UNMDP. Si bien Estados Unidos es el país con el que se establecen el mayor número de colaboraciones, la presencia de España, Brasil y México imprime un fuerte sesgo iberoamericano a este proceso. Aun así, y desde una mirada más estrictamente regional, Latinoamérica no constituye el principal destino de las colaboraciones, ya que sumando al resto de los países que figuran en el listado como Chile, Uruguay, Colombia y Venezuela alcanzan un volumen total de producción del $26 \%$. Siguiendo esta línea de agrupamiento por bloque geográfico, el conjunto de países con mayor gravitación lo constituyen aquellos pertenecientes a la Unión Europea, que concentran el 38\% de la producción colaborada, destacándose principalmente España, Alemania y Francia. Finalmente, el bloque de la América anglosajona, con Estados Unidos y Canadá, se posiciona como el tercer espacio en importancia con el $20 \%$ de las publicaciones. Esta vía de análisis basada en una clasificación por bloques geográficos es, en principio, la única posible de acuerdo a los datos disponibles aunque las razones de la elección e intensidad de las relaciones responden, desde el contexto institucional, a un conjunto de variables más específicas que van desde lo estrictamente disciplinar hasta cuestiones de política científica. No obstante esta limitación, puede afirmarse que el mayor número de colaboraciones se establecen con aquellos países que ostentan los sistemas científicos más desarrollados dentro de cada bloque. 
Tabla 2. Países con mayor peso en la producción colaborada internacionalmente

\begin{tabular}{|c|c|c|c|}
\hline Países & Colaboraciones & $\%$ & $\%$ acum. \\
\hline Estados Unidos & 689 & 16,27 & 16,27 \\
\hline España & 615 & 14,52 & 30,79 \\
\hline Brasil & 468 & 11,05 & 41,84 \\
\hline Alemania & 256 & 6,04 & 47,88 \\
\hline México & 191 & 4,51 & 52,39 \\
\hline Francia & 187 & 4,41 & 56,80 \\
\hline Chile & 178 & 4,20 & 61,01 \\
\hline Italia & 154 & 3,64 & 64,64 \\
\hline Canadá & 153 & 3,61 & 68,25 \\
\hline Reino Unido & 135 & 3,19 & 71,44 \\
\hline Uruguay & 127 & 3,00 & 74,44 \\
\hline Australia & 103 & 2,43 & 76,87 \\
\hline Colombia & 71 & 1,68 & 78,55 \\
\hline Países Bajos & 60 & 1,42 & 79,96 \\
\hline Venezuela & 51 & 1,20 & 81,17 \\
\hline Suiza & 48 & 1,13 & 82,30 \\
\hline China & 48 & 1,13 & 83,43 \\
\hline Polonia & 47 & 1,11 & 84,54 \\
\hline Portugal & 43 & 1,02 & 85,56 \\
\hline Japón & 41 & 0,97 & 86,52 \\
\hline Israel & 39 & 0,92 & 87,45 \\
\hline Rusia & 36 & 0,85 & 88,30 \\
\hline Nueva Zelanda & 35 & 0,83 & 89,12 \\
\hline Bélgica & 34 & 0,80 & 89,92 \\
\hline Suecia & 30 & 0,71 & 90,63 \\
\hline$\ldots$ & $\ldots$ & $\ldots$ & $\ldots$ \\
\hline
\end{tabular}

Fuente: elaboración propia

Tabla 2. Países con mayor peso en la producción colaborada internacionalmente

Cuando desagregamos los países con mayor presencia en la colaboración por grandes áreas temáticas tomando como base la acumulación del 50\% de la producción se observa que, más allá de las diferentes posiciones y pequeñas variantes, el núcleo de cinco países mantiene la centralidad (Tabla 3). En este agrupamiento se suma Chile como uno de los países que también mantiene uno de los flujos de intercambio más importante a nivel regional. En el área Social Sciences la presencia por demás destacada de España obedece fundamentalmente a la cuestión idiomática, ya que el total de publicaciones colaboradas con autores de este país está publicada en lengua española. 
Tabla 3. Distribución de los países con mayor colaboración por área temática

\begin{tabular}{|c|c|c|c|c|}
\hline Área temática & País & Colaboraciones & $\%$ & $\%$ acum. \\
\hline \multirow{5}{*}{ Health Sciences } & Estados Unidos & 75 & 14,34 & 14,34 \\
\hline & España & 61 & 11,66 & 26,00 \\
\hline & Brasil & 60 & 11,47 & 37,48 \\
\hline & Chile & 39 & 7,46 & 44,93 \\
\hline & Alemania & 23 & 4,40 & 49,33 \\
\hline \multirow{5}{*}{ Life Sciences } & Estados Unidos & 358 & 18,98 & 18,98 \\
\hline & Brasil & 194 & 10,29 & 29,27 \\
\hline & España & 167 & 8,85 & 38,12 \\
\hline & Alemania & 130 & 6,89 & 45,01 \\
\hline & Chile & 122 & 6,47 & 51,48 \\
\hline \multirow{5}{*}{ Physical Sciences } & España & 408 & 16,66 & 16,66 \\
\hline & Estados Unidos & 357 & 14,58 & 31,24 \\
\hline & Brasil & 290 & 11,84 & 43,08 \\
\hline & Alemania & 141 & 5,76 & 48,84 \\
\hline & Francia & 119 & 4,86 & 53,70 \\
\hline \multirow{5}{*}{ Social Sciences } & España & 62 & 24,03 & 24,03 \\
\hline & Estados Unidos & 30 & 11,63 & 35,66 \\
\hline & Brasil & 22 & 8,53 & 44,19 \\
\hline & México & 19 & 7,36 & 51,55 \\
\hline & Chile & 16 & 6,20 & 57,75 \\
\hline
\end{tabular}

Fuente: elaboración propia

Al momento de examinar las instituciones con las cuales la UNMDP colabora puede observarse un gran abanico conformado por 1320 entidades, distribuidas principalmente en universidades, centros de investigación, entidades gubernamentales, organizaciones privadas, entre otras. La dispersión es muy grande, aunque existe un grupo identificable a partir de la frecuencia de las colaboraciones realizadas y que guardan una relación directa de procedencia con el grupo de "países núcleo" (Tabla 4).

A partir de un análisis de co-ocurrencia de las instituciones extranjeras colaborantes pudo elaborarse un mapa por medio del análisis de redes sociales (ARS) al cual se aplicó el cálculo de modularidad para determinar la existencia de comunidades o clusters que pudieran aportar un nivel de mayor precisión al análisis. El resultado fue la obtención de seis agrupamientos con distintos grados de densidad e interacciones (Figura 3). Para una representación más ordenada y una mejor interpretación del grafo resultante se aplicó un umbral de visualización de al menos 20 colaboraciones. 
Tabla 4. Principales instituciones extranjeras colaborantes

\begin{tabular}{|l|l|c|}
\hline Instituciones extranjeras & País & Colaboraciones \\
\hline University of California & Estados Unidos & 103 \\
\hline French National Centre for Scientific Research & Francia & 95 \\
\hline Boston University & Estados Unidos & 86 \\
\hline Universidade de Sao Paulo & Brasil & 80 \\
\hline Universidad de la República & Uruguay & 75 \\
\hline Universidad del País Vasco & España & 58 \\
\hline National Research Council of Italy & Italia & 54 \\
\hline University of Minnesota & Estados Unidos & 54 \\
\hline Universidad de La Serena & Chile & 45 \\
\hline Helmholtz Association of German Research Centres & Alemania & 44 \\
\hline Universidad Nacional Autónoma de México & México & 44 \\
\hline Universidade Estadual Paulista & Brasil & 44 \\
\hline Auburn University & Estados Unidos & 40 \\
\hline National Institute of Applied Sciences of Lyon & Francia & 40 \\
\hline University of Nebraska & Estados Unidos & 40 \\
\hline University of Washington & Estados Unidos & 40 \\
\hline Universidad de Concepción & Chile & 39 \\
\hline Universidade Federal do Paraná & Brasil & 39 \\
\hline Universidad de Barcelona & España & 33 \\
\hline$\ldots$ & $\ldots$ & $\ldots$ \\
\hline
\end{tabular}

En el grafo pueden observarse las comunidades delimitadas por color y la importancia de las instituciones (número de colaboraciones) por el tamaño de su nombre. Esencialmente, lo que se aprecia a primera vista son las principales redes de colaboración institucionales con las que la UNMDP desarrolla o ha desarrollado algún tipo de vinculación científica a nivel internacional. Como primer análisis, puede decirse que el grueso de interacciones con instituciones extranjeras se establece principalmente con aquellas provenientes de países latinoamericanos, Estados Unidos y los países de Europa Central. Al mismo tiempo, la conformación de los clusters no responde necesariamente a un patrón disciplinar o temático ya que en ellos conviven procesos de colaboración provenientes de diferentes campos científicos.

En orden de importancia o densidad se destaca el agrupamiento color verde conformado en su mayor parte por universidades estadounidenses, lideradas por la University of California, University of Colorado, University of Washington y la Boston University. En segundo lugar de importancia se ubica la subred celeste en donde se destacan el Consejo Superior de Investigaciones Científicas (CSIC) de España y la Universidad Nacional Autónoma de México como las dos instituciones más gravitantes vinculadas a otras universidades españolas y latinoamericanas importantes. En tercer orden, en color violeta, aparece un conglomerado de corte latinoamericano constituido mayoritariamente por universidades brasileñas con la Universidade de Sao Paulo como actor principal, vinculada fuertemente a la Universidad de la República del Uruguay y la Universidad de Concepción de Chile, entre otras. En cuarto lugar 


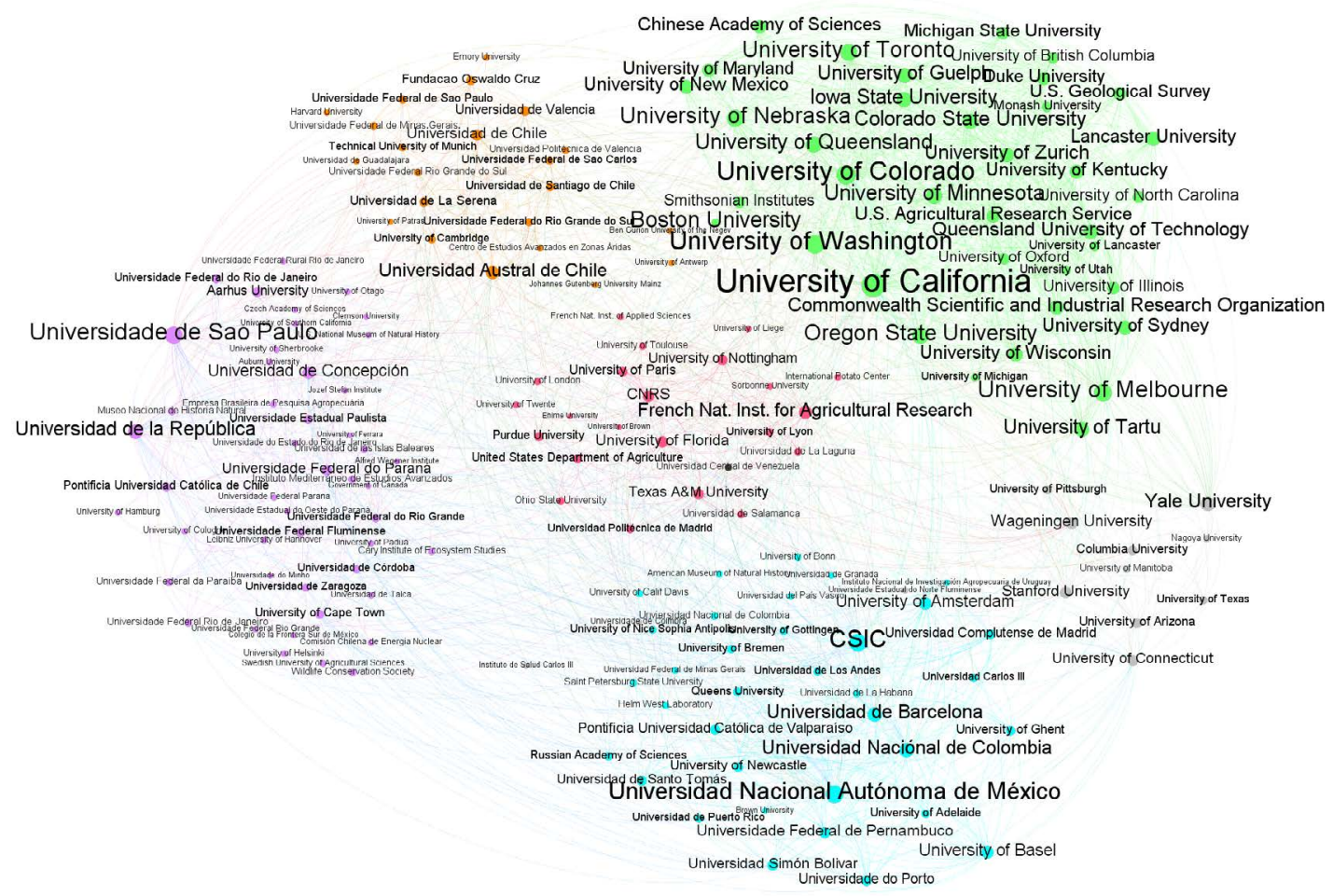

Figura 3. Principales comunidades de instituciones en relación al número de colaboraciones establecidas (umbral $\geq 20$ colaboraciones).

y delimitada por el color anaranjado se ubica un agrupamiento estructurado principalmente en torno a cuatro grandes instituciones chilenas como son la Universidad Austral de Chile, Universidad de Chile, Universidad de La Serena y la Universidad de Santiago de Chile. En quinto orden y en el centro del grafo en color rojo se agrupan un conjunto de instituciones cuyo peso se reparte entre tres entidades francesas de mucho prestigio como son el Centre national de la recherche scientifique (CNRS), el French National Institute for Agricultural Research y la University of Paris, y un conjunto de instituciones estadounidenses entre las que se destacan la University of Florida, el Department of Agriculture y la Texas A\&M University. Finalmente, aparece un pequeño agrupamiento constituido por universidades de élite estadounidenses Stanford, Yale, Columbia, Connecticut y Pittsburgh, de Japón Nagoya University y de los Países Bajos como la Wageningen University.

$\mathrm{Al}$ analizar el componente liderazgo científico de la UNMDP en torno a la producción científica con colaboración extranjera $(\mathrm{N}=3417)$ puede apreciarse que la institución obtiene un buen desempeño ya que en el $53 \%(\mathrm{n}=1803)$ de los artículos aparece como entidad responsable de la investigación publicada. Este aspecto resulta de enorme relevancia ya que pone de manifiesto las capacidades científicas existentes y el modo en que la Universidad se vincula en los procesos de internacionalización de la investigación. El cálculo de esta variable se completa con el $8 \%(n=274)$ de publicaciones lideradas por otras instituciones nacionales y el $39 \%(n=1340)$ restante por entidades de países extranjeros. Al hacer foco en los países de filiación de la producción liderada por instituciones extranjeras se observa nuevamente una correspondencia con el grupo de "países núcleo" con los que existe mayor flujo de colaboración (Figura 4). 


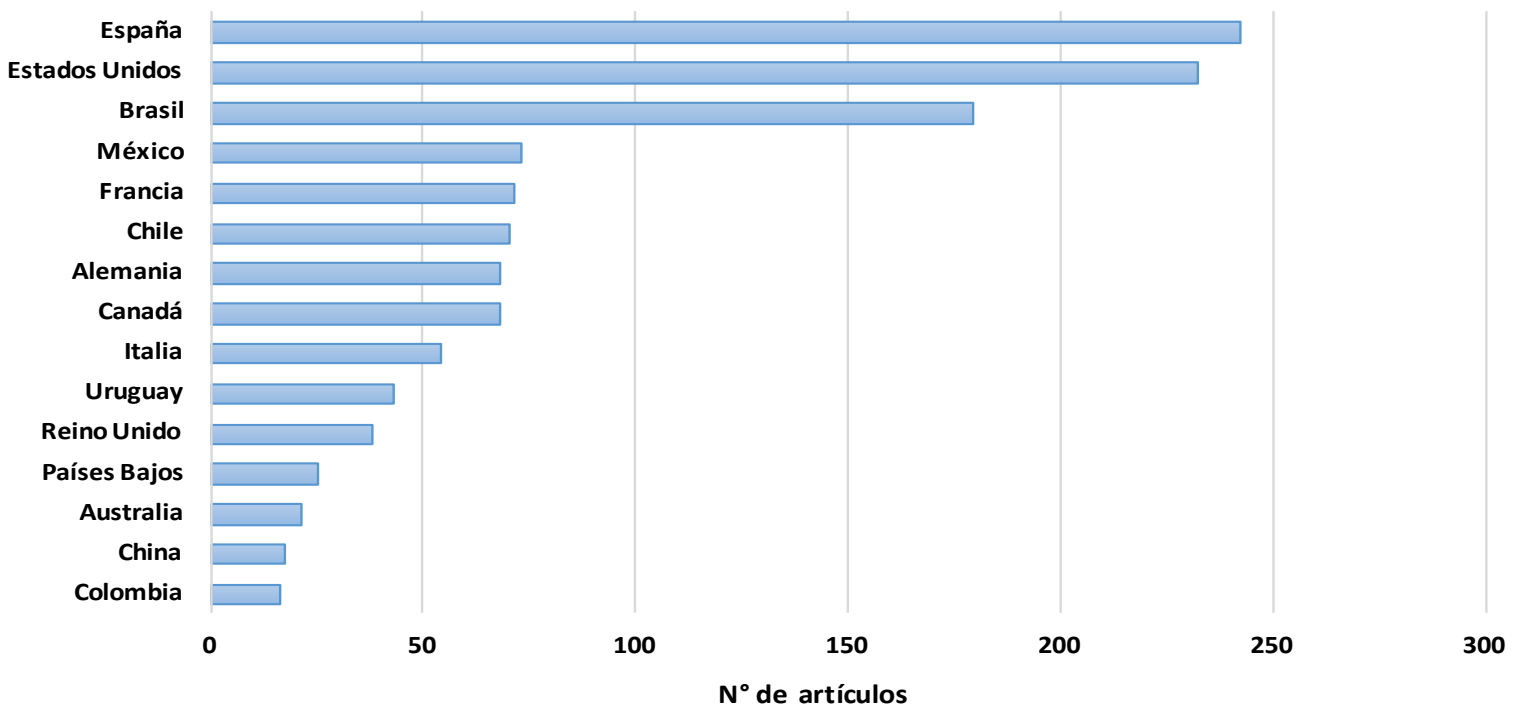

Fuente: elaboración propia

Figura 4. Países con mayor influencia en el liderazgo de la producción colaborada internacionalmente expresada en número de artículos

De los 50 países que ejercen liderazgo en los trabajos publicados, la lista presentada en la figura anterior (16 países) representa el $90 \%$ de la producción científica delimitada dentro de este indicador. Cuando se observa la distribución de esta variable dentro de las grandes áreas temáticas puede apreciarse que las disciplinas ligadas a las Physical Sciences (52\%) y a las Life Sciences (35\%) son las que tienen mayor dependencia de proyectos cuyas direcciones o responsabilidades recaen en investigadores extranjeros. Dado el enorme peso que tienen estas dos áreas en el análisis global de la producción estudiada puede inferirse que esta aparente dependencia es, en realidad, producto de la enorme dinámica que atraviesan estos campos y la existencia de una mayor tradición de vinculación en proyectos colaborativos internacionales.

Por último, el cálculo del indicador idioma de publicación arroja resultados previsibles en términos de la hegemonía del idioma inglés alcanzando, en aquellas contribuciones con colaboración internacional, al 96\% de los artículos publicados (Figura 5). Esta realidad se replica, con valores similares, tanto en las publicaciones colaboradas a nivel nacional como en el total de la producción en colaboración de la UNMDP.La segunda lengua de publicación es el español aunque en valores marginales, representando sólo el 3,5\% de las publicaciones con colaboración internacional, porción que se sitúa, casi en su totalidad (80\%), en los artículos pertenecientes al área Social Sciences. Estas distribuciones son consistentes con el sesgo idiomático subyacente en las publicaciones de la corriente principal, en donde la lengua inglesa posee una marcada preeminencia. También se encuentra en línea con las culturas de publicación de las dos áreas de mayor peso en este estudio, Physical Sciences y Life Sciences, que comunican casi la totalidad de su producción a través de las revistas indizadas en estas bases de datos. 


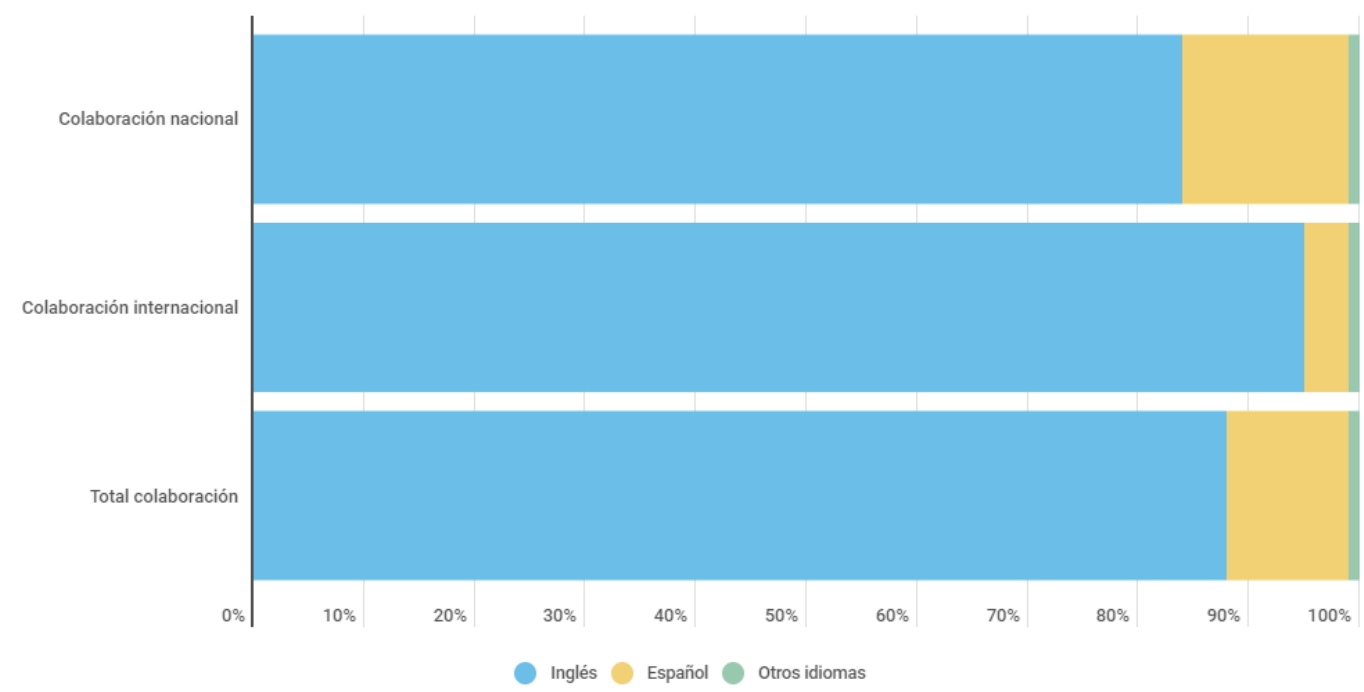

Figura 5. Distribución porcentual de los artículos por idioma de publicación

\section{Conclusiones}

El análisis de la internacionalización de la ciencia y su descripción desde el contexto de una universidad latinoamericana permite pensar diversas cuestiones relacionadas con la influencia que puede tener tal fenómeno en la cultura científica de una institución y su desarrollo. Argentina integra el grupo de países latinoamericanos, junto con Brasil, Chile, México y Colombia, que mayor colaboración tienen con regiones centrales o de mayor desarrollo científico debido, en gran medida, a programas específicos que sobre inicios de este siglo se impulsaron y financiaron desde Europa y otras regiones centrales. La UNMDP se inscribe en esta tendencia de apertura internacional, en términos de la colaboración científica y coautoría, presentando valores en constante crecimiento en las últimas décadas. Los resultados aquí mostrados abonan esta afirmación.

En términos históricos, del total de la producción científica colaborada de la UNMDP localizada en la corriente principal el $38 \%$ ha sido con participación internacional, aunque esta variable debe observarse en su evolución, llegando en la actualidad a representar casi la mitad de sus publicaciones. Si bien este fenómeno se manifiesta en todas las áreas de conocimiento, es importante resaltar la dificultad de una división estricta en este sentido habida cuenta del proceso de asignación de las categorías temáticas por las bases de datos utilizadas que, además de poseer criterios diferentes, poseen un gran solapamiento producto de la naturaleza interdisciplinaria de la investigación publicada. Este aspecto explica, en parte, el comportamiento del área Health Sciences ya que, a pesar de presentar el menor volumen de producción del conjunto, supera en gran medida los porcentajes de colaboración internacional de las demás áreas, dando así la idea de que no es posible, en función de los datos empíricos aquí trabajados, establecer una relación directa entre el volumen de publicación y la capacidad de colaboración internacional. 
En el análisis de la coautoría en las publicaciones se verifica el cambio de comportamiento cuando estas se generan con participación de autores extranjeros, lo cual refuerza la noción de que las culturas de publicación locales, en un contexto de colaboración con otros países, viran hacia la multiautoría. Este aspecto se expresa con mucha notoriedad en el área Social Sciences.

En el terreno de las instituciones y países extranjeros involucrados en la producción colaborada de la UNMDP se comprobó que, más allá del número contabilizado, el grueso de la producción (50\%) se circunscribe a tan sólo 5 países, de los que se desprenden un número igualmente acotado de instituciones. De los datos mostrados se observa que la Universidad posee lazos de colaboración consolidados con un grupo selecto de universidades e institutos de investigación de Estados Unidos, Latinoamérica (Brasil, Chile, México en particular) y Europa (España, Alemania, Francia).

Más de la mitad de la producción colaborada a nivel internacional fue liderada por la UNMDP, permitiendo concluir que las capacidades científicas locales poseen relevancia mundial. En particular, este indicador permitió comprobar la consolidación y la posición de centralidad que ostentan las áreas Physical Sciences y Life Sciences, que por volumen de producción y cobertura disciplinar son aquellas en las que el proceso de internacionalización se hace más evidente.

Es de suponer que el fenómeno de la internacionalización en la UNMDP medido en base a indicadores de producción continúe con un incremento sostenido aunque bajo diferentes velocidades según el área temática que se tome. En este sentido, no es ajeno el hecho de que esta investigación está basada en datos provenientes del mainstream en donde las ciencias sociales y humanidades se encuentran subrepresentadas en términos de su caudal productivo y en los circuitos de circulación habitualmente utilizados, por fuera de las revistas indizadas en estas dos bases de datos. Aun así, la investigación ha pretendido mostrar, desde una mirada amplia, las características estructurales del fenómeno de la internacionalización en esta institución a partir de indicadores que aporten los principales rasgos de dicha dimensión. Será necesario, en este sentido, profundizar el análisis en aquellos aspectos institucionales y sectoriales que permitan explicar, con mayor precisión, los factores que inciden y modelan este proceso. 


\section{Q Referencias bibliográficas}

》 Beaver, D. y R. Rosen. 1979. Studies in Scientific Collaboration. Part III. Professionalization and the natural history of modern scientific coautorship. En Scientometrics. Vol. 1, no. 3, 231-245. <http://dx.doi.org/10.1007/BFo2016308>

"Beigel, María Fernanda. 2013. Centro y periferias en la circulación internacional del conocimiento. En Nueva Sociedad. No. 245, 110-123. <https://ri.conicet.gov. ar/handle/11336/1232> [Consulta: 3 marzo 2021].

»Conferencia Regional de Educación Superior. 2008. CRES 2008: declaración y plan de acción de la Conferencia regional de Educación Superior en América Latina y el Caribe. Cartagena: UNESCO-IESALC.

"De Filippo, Daniela, Sergio Marugán y Elías Sanz-Casado. 2014. Perfil de colaboración científica del sistema español de educación superior. Análisis de las publicaciones en Web of Science (2002-2011). Revista Española de Documentación Científica. Vol. 37, no. 4, e067. <http://dx.doi.org/10.3989/redc.2014.4.1155>

"Diker, Gabriela. 2018. Internacionalizar la educación superior: con quién, para qué, cómo. En Balance y desafíos hacia la CRES 2018. Ciudad Autónoma de Buenos Aires: IEC-CONADU, p. 151-156.

»Escribà-Sales, Eudald y Sergi Cortiñas. 2013. La internacionalización y las coautorías en las principales revistas científicas de Comunicación en España. En Comunicar. Vol. 41, no. 21, 35-44. <https://doi.org/10.3916/C41-2013-03>

»Jeremić, Veljko, Marina Jovanović-Milenković, Zoran Radojičić y Milan Martić. 2013. Excellence with leadership: the crown indicator of SCImago Institutions Rankings Iber report. En El profesional de la información. Vol. 22, no.5, 474-480. <http://dx.doi.org/10.3145/epi.2013.sep.13> [Consulta: 3 marzo 2021].

» Jung, Nina y Alejandro Arnulfo Ruiz-León. 2018. Lo local y lo global de la colaboración científica: ¿qué significa, y cómo visualizarlo y medirlo? En Revista Española de Documentación Científica. Vol. 41, no. 2, e203. <https://doi.org/10.3989/ redc.2018.2.1463>

» Katz, J. Sylvan y Ben R. Martin. 1997. What is research collaboration? En Research Policy. Vol. 26, 1-18. <https://doi.org/10.1016/Soo48-7333(96)oo917-1>

» Laclette, Juan Pedro, Patricia Zúñiga-Bello y Cristina Puga-Espinosa, eds. 2011. Informe sobre las ciencias sociales en el mundo: las brechas del conocimiento. México: Unesco/Foro Consultivo Científico y Tecnológico. <http://www.foroconsultivo.org.mx/libros_editados/informe_sobre_las_ciencias_sociales_en_el_ mundo.pdf> [Consulta: 3 marzo 2021].

» Leydesdorff, Loet, Caroline S. Wagner, Han-Woo. Park y Jonathan Adams. 2013. Colaboración internacional en ciencia: mapa global y red. En El profesional de la información. Vol. 22, no. 1, 87-94. <https://recyt.fecyt.es/index.php/EPI/article/ view/33263/17838> [Consulta: 3 marzo 2021].

"López-Robles, José Ricardo, Javier Guallar, Manuel Jesús Cobo, José Ramón Otegi-Olaso y Nadia Karina Gamboa-Rosales. 2019. La internacionalización de El Profesional de la Información (EPI) en el último lustro (2013-2018). Trabajo presentado al X Conferencia internacional sobre revistas científicas CRECS, realizado en Guadalajara (México) del 23-24 de octubre de 2019. 
»Michelini, Gabriela. 2018. Revisión comparativa de instrumentos para evaluar la internacionalización de la ciencia en el sistema universitario. En Revista lberoamericana de Ciencia, Tecnología y Sociedad. Vol. 37, no. 13, 233-256.

»Mindeli, L. E. y V. A. Markusova. 2015. Bibliometric Studies of Scientific Collaboration: International Trends. En Automatic Documentation and Mathematical Linguistics. Vol. 49, no. 2, 59-64. <https://doi.org/10.3103/Sooo5105515020065>

»Moed, Henk F. 2017. Applied Evaluative Informetrics: Qualitative and Quantitative Analysis of Scientific and Scholarly Communication. Cham: Springer International Publishing.

»Mosbah-Natanson, Sébastien e Yves Gingras. 2014. The globalization of social sciences? Evidence from a quantitative analysis of 30 years of production, collaboration and citations in the social science: (1980-2009). En Current Sociology. Vol. 62, no.5, 626-646. <https://doi.org/10.1177/0011392113498866>

» Moya-Anegón, Félix. 2012. Liderazgo y excelencia de la ciencia española. En El profesional de la información. Vol 21, no. 2, 125-128. <http://dx.doi.org/10.3145/ epi.2012.mar.01>

» Navas-Fernández, Miguel, Ernest Abadal y Rosângela S. Rodrigues. 2018. Internationality of Spanish scholarly journals indexed in Web of Science and Scopus. En Revista Española de Documentación Científica. Vol. 41, no. 3, e209. <https:// doi.org/10.3989/redc.2018.3.1498 >

" Oliveira, Giulia Xisto de, Debora Vestena, Carlos Rafael Röhrig da Costa, Luciana Davi Traverso y Roberto Schoproni Bichueti. 2020. Internacionalização das universidades: estudo sobre a produção científica. En Gestão e Desenvolvimento. Vol. 17, no. 1, 196-217. <https://doi.org/10.25112/rgd.v17i1.2020>

" Organización para la Cooperación y el Desarrollo Económicos. 2015. Frascati Manual 2015. Guidelines for Collecting and Reporting Data on Research and Experimental Development. The Measurement of Scientific, Technological and Innovation Activities. París: OCDE. <http://dx.doi.org/10.1787/9789264239012-en>

"Pandiella, Andrés, Carlos García-Zorita y Elías Sanz-Casado. 2019. Análisis de la internacionalización de la Revista Española de Documentación Científica: 2010-2015. En Revista Española de Documentación Científica. Vol. 42, no. 1, e223. <https://doi.org/10.3989/redc.2019.1.1594 >

»Red Iberoamericana de Indicadores de Ciencia y Tecnología. 2007. Manual de Indicadores de Internacionalización de la Ciencia y la Tecnología, Manual de Santiago. Buenos Aires: RICYT.

»Robinson-García, Nicolas y Ismael Ràfols, I. 2020. The differing meanings of indicators under different policy contexts. The case of internationalisation. En Cinzia Daraio, Wolfgang Glänzel, ed. Evaluative Informetrics: The Art of Metrics-Based Research Assessment: Festschrift in Honour of Henk F. Moed, p. 213-232. Cham: Springer International Publishing.

»Robinson-García, Nicolas y Carlos B. Amat. 2018. ¿Tiene sentido limitar la coautoría científica? No existe inflación de autores en Ciencias Sociales y Educación en España. En Revista Española de Documentación Científica. Vol. 41, no. 2, e201. <https://doi.org/10.3989/redc.2018.2.1499>

» Schubert, András y Gábor Schubert. 2020. Internationality at university level. En Scientometrics. Vol. 123, no. 3, 1341-1364. <https://doi.org/10.1007/s11192-02003443-3> 
» Sebastián, Jesús. 2010. El Manual de Santiago: una guía para medir la internacionalización de la i+d. En Red Iberoamericana de Indicadores de Ciencia y Tecnología (RICYT). El Estado de la Ciencia. Principales Indicadores de Ciencia y Tecnología Iberoamericanos/Interamericanos 2007. Buenos Aires: RICYT, p. 47-53.

»Sebastián, Jesús. 2011. Dimensiones y métrica de la internacionalización de las universidades. En Universidades, no. 51, 3-16. <http://www.redalyc.org/articulo. oa? id=37322089002> [Consulta: 3 marzo 2021].

»Sleimen, Silvia. 2015. Producción científica de la ciudad de Mar del Plata (Argentina) en Web of Science: 1975-2012. Madrid: UC3M, 2015. 504 p. Tesis doctoral.

"Sonnenwald, Diane H. 2007. Scientific collaboration. En Annual Review of Information, Science and Technology. Vol. 41, no. 1, 643-68o. <https://doi.org/10.1002/ aris.2007.1440410121>

»Souza, Cláudia Daniele de, Daniela De Filippo y Elías Sanz-Casado. 2019. El papel de la internacionalización de la educación superior en la producción científica brasileña. En Ensaio: Avaliação e Políticas Públicas em Educação. Vol. 26. <http://dx.doi.org/10.159o/s0104-40362019002701721> 
